\section{Genomewide vs. genome dive}

\author{
By Steven Edelson, Executive Editor \& \\ Lev Osherovich, Senior Writer
}

A series of commentaries in the New England Journal of Medicine has painted a dichotomy between the utility of both genomewide association studies and deep sequencing of individuals, which the general media has reported as an expert view that the value of GWA research has been exhausted.

A commentary by Duke University's David Goldstein made the case for shifting resources away from GWA studies and toward deep sequencing. Although he acknowledged that GWA studies have found common SNP variants associated with a given disease, he argued that "common variation is packing much less of a phenotypic punch than expected."

Goldstein is director of the Center for Population Genomics \& Pharmacogenetics at the university's Institute for Genome Sciences \& Policy. His views got prominent billing in The New York
"In pointing at everything, geneticists would point at nothing."

-David Goldstein, Duke University studies will continue to be used initially to identify disease-related pathways and networks, followed by deep sequencing of individual's genes to find very rare variants within those networks that may have a causative link to the disease or may carry significant predictive power.

\section{Limits of GWA}

One critique of GWA studies is that many of the common SNPs associated with disease typically increase the risk of the condition by only a couple of percentage points. ${ }^{5}$ Thus, Goldstein argued, one would potentially need to identify a high number of SNPs, covering a broad swath of the genome, to fully explain a disease's genetic underpinnings.

But, "in pointing at everything, geneticists would point at nothing," he wrote. "If common variants are responsible for most genetic components of type 2 diabetes, height and similar traits, then genetics would provide relatively little guidance about the biology of these conditions, because most genes are 'height genes' or 'type 2 diabetes genes.'

Instead, Goldstein wrote, "most genetic control is due to rarer variants, either single-site or structural, that are not represented in the current studies and that have considerably larger effects than common variants."

According to Goldstein, initial GWA studies for a given disease are worth doing, as those are likely to find the common SNP variants most strongly associated with a disease. However, he argued that any larger follow-up studies would yield dramatically diminishing returns.

"It's hard to have any enthusiasm for conducting genome scans with the use of ever larger Times, which called the debate about the merits of GWA studies versus those of sequencing for rare variants an "unexpected impasse."

According to the Times, Goldstein "said resources should be switched away from these highly expensive studies, which in his view have now done their job."

GWA approaches also were targeted in a related commentary in NEJM by Peter Kraft and David Hunter of the Harvard School of Public Health, who argued that it is generally premature for companies to sell genetic risk prediction kits, which use data from GWA studies, directly to patients. ${ }^{3}$ "We are still too early in the cycle of discovery for most tests that are based on newly discovered associations to provide stable estimates of genetic risk for many diseases," they wrote.

Kraft is an associate professor of epidemiology and biostatistics at the school, whereas Hunter is a professor in the departments of epidemiology and nutrition.

In a counterpoint perspective, Joel Hirschhorn from Children's Hospital Boston argued that GWA studies have hardly run their course. " The main goal of these studies is not prediction of individual risk but rather the discovery of biologic pathways underlying polygenic diseases and traits," he wrote. "It is already clear that the genes being identified expose relevant biology."

The reality, according to researchers contacted by SciBX, is that both approaches will remain important. According to this point of view, GWA cohorts after a study of the first several thousand subjects has identified the strongest determinants among common variants," Goldstein wrote.

As a result, Goldstein urged a shift "from genome scans of ever larger samples to studies of rarer variants of larger effect."

In his counterpoint arguing the relevance of common GWA hits for determining the genetic basis of a trait or disease, Hirschhorn described a recent study showing that 11 of 23 loci associated with variation in lipid levels encode enzymes already known to influence lipid metabolism. ${ }^{6}$ According to Hirschhorn, this implies that the other 12 proteins could also contribute to disease and represent drug targets.

Thus, instead of shifting focus away from GWA studies, Hirschhorn suggested that researchers "should strive for as complete a catalogue of validated risk variants as possible, through additional genomewide association studies and complementary approaches."

Hirschhorn is an associate professor of genetics at Children's Hospital Boston.

\section{Passing the impasse}

Rather than stopping GWA work, researchers contacted by SciBX argued that these kinds of studies will remain an important first step for multiple diseases.

"There is no 'dichotomy', just two complementary approaches," Hirschhorn told SciBX. "Indeed, they are likely to be synergistic-one 
can sequence the genes at loci that come up in association studies and get confirmatory association evidence for genes that appear to have an excess of rare variants but where the evidence is not sufficiently strong."

"I see geneticists on a bullet train—not at an impasse," said David Altshuler, professor of genetics and medicine at Harvard Medical School and director of the Broad Institute of MIT and Harvard's Program in Medical and Population Genetics. "There was never any suggestion that GWA studies were a destination as opposed to a stop along the way."

Altshuler also thinks deep sequencing is the logical next step in areas where there has been a deluge of GWA studies, and his own lab is a case in point. After about a decade of chasing SNPs by means of GWA studies, "we've been focused on sequencing for the last year or two-but in the regions we found via GWA studies."

Teri Manolio, director of the Office of Population Genomics at the NIH, agreed. "We shouldn't give up on genomewide association studies-there's still a lot they can tell us and there are lots of diseases that haven't even been studied," she said.

Mark McCarthy, professor of diabetic medicine at the University of Oxford, is also thinking along similar lines. "I agree that we should be looking at rare variants, but I have a hard time accepting that GWA studies are a waste of time because they have only found about $20 \%$ of the variation" relevant to disease in humans, he said.

McCarthy disagreed with Goldstein's assertion that common variants may lack utility because they implicate numerous genes in complex diseases such as diabetes.

"Pharma wants to understand each of the genes, to see if it's too little or too much," McCarthy said. "Pharma should be interested in those targets even if there's another 100 genes that also have effects. It doesn't matter if there are 10 genes in the same pathway that are implicated in diabetes risk. In fact, this would be an encouraging finding that implicates the pathway."

McCarthy added that genes fingered by GWA studies may still play a strong role in disease even if common variants in the genes contribute only a little to overall disease risk. This is because natural selection weeds out extreme mutations with profound disease-related effects but may spare mutations in the very same genes that cause more modest effects.

Thus, he said, even low-impact GWA hits can be a foot in the door to understanding fundamental disease processes.

Indeed, the ability of GWA studies to tease out biological networks in a disease may be their most important offering, according to Eric Schadt, VP and CSO of Sage.

"There are perhaps hundreds or even thousands of genes that are subtly perturbed by variations in DNA that go on to increase/decrease risk of disease or affect disease progression," he said. "These genes are not random with respect to the biological processes that affect disease but are instead organized into coherent networks, so that if you are able to identify the molecular network that captures the different perturbations, then you can really refine that given subtype of disease and get to a model that is predictive."

Sage is a not-for-profit genomics institute that is being spun out of Merck \& Co. Inc.'s Rosetta Inpharmatics Inc. unit.

\section{Peering forward}

With a broad consensus on the complementarity of GWA studies and deep sequencing - the latter being seen as the logical progression from the former-deciding which individuals to deep sequence remains an open question.

According to Manolio, GWA studies are likely to provide clues about whom to sequence. "The initial GWA study could isolate interesting variants, and from there functional studies could follow," she said.

She added that one approach could be to take patients "who have extreme phenotypes. For example, you might look at people who get diabetes when they're very young or when they're lean. You could also look at patients with diabetes that is hard to control or has complications."

Manolio concluded: "You want to sequence the people who are potentially the most informative. But nobody really knows who that is, and we want to be open to multiple options."

In addition to the research utility offered by both GWA and deep sequencing, Altshuler thinks both approaches can also address big markets. "The only thing that matters to the commercial sector is whether there's a clinical market," he said. "The question is will sequencing be used in clinical medicine-yes. Will genotyping be used-yes, as well."

"Both ways can get to new biology, which is the main goal," Hirschhorn, told SciBX.

Jay Kaufman, VP of product marketing at Affymetrix Inc., added that GWA studies enable other approaches to hunting for disease genes and that deep sequencing is not the only alternative to more GWA analysis.

"Many people contend that GWA studies are not going away in the near term," he said. "However, many of the same people also suggest that looking at other types of variation, whether it be minor allele frequency SNPs, insertions and deletions, structural variants or other yet untapped sources of genetic variation, is the next step in an ongoing process."

Affymetrix markets a range of DNA microarray products for diagnostics and research. According to Kaufman, increasingly high-resolution GWA studies have already had the beneficial effect of adding more SNPs to the public domain, providing "a useful basis for designing array products to enable cost-effective and focused study of rare and structural variants."

Goldstein did not provide additional comments to SciBX.

Edelson, S. \& Osherovich, L. SciBX 2(16); doi:10.1038/scibx.2009.646 Published online April 23, 2009

\section{REFERENCES}

1. Goldstein, D. N. Engl. J. Med.; published online April 15, 2009; doi:10.1056/NEJMp0806284

2. Wade, N. Genes show limited value in predicting diseases. The New York Times A1 (April 16, 2009)

3. Kraft, P. \& Hunter, D. N. Engl. J. Med.; published online April 15, 2009; doi:10.1056/NEJMp0810107

4. Hirschhorn, J. N. Engl. J. Med.; published online April 15, 2009; doi:10.1056/NEJMp0808934

5. McCarthy, M.I. et al. Nat. Rev. Genet. 9, 356-369 (2008)

6. Molhke, K.L. et al. Hum. Mol. Genet. 17, R102-R108 (2008)

COMPANIES AND INSTITUTIONS MENTIONED

Affymetrix Inc. (NASDAQ:AFFX), Santa Clara, Calif.

Broad Institute of MIT and Harvard, Cambridge, Mass.

Children's Hospital Boston, Boston, Mass.

Duke University, Durham, N.C.

Harvard Medical School, Boston, Mass.

Harvard School of Public Health, Boston, Mass.

Merck \& Co. Inc. (NYSE:MRK), Whitehouse Station, N.J.

National Institutes of Health, Bethesda, Md.

Rosetta Inpharmatics Inc. (NASDAQ:RSTA), Kirkland, Wash.

Sage, Seattle, Wash.

University of Oxford, Oxford, U.K. 